\title{
Quadrature Formulas for Functions with Poles Near the Interval of Integration*
}

\author{
By Giovanni Monegato
}

\begin{abstract}
In this paper, we examine the construction of quadrature rules of interpolatory type, using only real function values, for functions with complex conjugate pairs of poles near the interval of integration.
\end{abstract}

1. Introduction. In some recent papers, Elliott and Paget [3], Sloan and Smith [14]-[19], and Smith, Sloan and Opie [20], have examined properties of the so-called product integration rules of interpolatory type, i.e., quadrature formulas of the form

$$
\int_{a}^{b} k(x) f(x) d x \doteq \sum_{j=1}^{n} w_{n j}(k) f\left(x_{n j}\right)
$$

with

$$
w_{n j}(k)=\int_{a}^{b} \frac{k(x) P_{n}(x)}{\left(x-x_{n j}\right) P_{n}^{\prime}\left(x_{n j}\right)} d x, \quad P_{n}(x)=k_{n} \prod_{i=1}^{n}\left(x-x_{n i}\right),
$$

obtained by preassigning a set of $n$ distinct points $\left\{x_{n j}\right\}$ in $(a, b)$, and determining the weights $\left\{w_{n j}(k)\right\}$ by requiring the rule to be exact whenever $f(x)$ is a polynomial of degree less than $n$. Integrals of the form (1) arise in many applications, particularly in integral equations.

Although the idea of constructing formulas of this type is very appealing, in practice it can be realized only for particular "kernel" functions $k(x)$. Indeed, the expressions given in the papers mentioned above for the computation of the weights $w_{n j}(k)$ require knowledge of the so-called modified moments

$$
m_{i}=\int_{a}^{b} k(x) P_{i}(x) d x, \quad i=0,1, \ldots,
$$

where $\left\{P_{i}(x)\right\}$ denotes a sequence of polynomials (usually orthogonal), and the zeros of $P_{n}(x)$ are precisely the nodes $\left\{x_{n j}\right\}$ of (1).

In their papers, Sloan and Smith have studied the convergence of product integration rules for several choices of the nodes $\left\{x_{n j}\right\}$, and under mild hypotheses on the functions $k(x)$ and $f(x)$. Here we report one of their main results (see [16], [18]).

Received October 17, 1984; revised July 29, 1985.

1980 Mathematics Subject Classification. Primary 65D32; Secondary 33A65.

*Work sponsored by the Italian Ministry of Education. 
THEOREM 1. Let $(a, b)=(-1,1)$ and $P_{n}(x)$ be the nth-degree polynomial orthogonal on $(-1,1)$ with respect to a nonnegative weight function $w(x)$, with zeros $x_{n 1}$, $x_{n 2}, \ldots, x_{n n}$, and (i)

$$
\int_{-1}^{1} \frac{|k(x)|^{2}}{w(x)} d x<\infty
$$

or (ii), $w(x)=u(x)(1-x)^{\alpha}(1+x)^{\beta}, u(x)$ positive in $[-1,1]$ and $u \in \operatorname{Lip}[-1,1]$, and

$$
\int_{-1}^{1}\left|k(x)(1-x)^{-\max [(2 \alpha+1) / 4,0]}(1+x)^{-\max [(2 \beta+1) / 4,0]}\right|^{p} d x<\infty
$$

for some real $p>1$.

Or (iii), let $x_{n j}=\cos ((2 j-1) \pi / 2 n)$ or $x_{n j}=\cos ((j-1) \pi /(n-1))$ or $x_{n j}=$ $\cos ((2 j-2) \pi /(2 n-1))$, and

$$
\int_{-1}^{1}|k(x)|^{p} d x<\infty
$$

for some real $p>1$.

Then, for all bounded Riemann integrable functions $f(x)$ we have

$$
\lim _{n \rightarrow \infty} \sum_{j=1}^{n} w_{n j}(k) f\left(x_{n j}\right)=\int_{-1}^{1} k(x) f(x) d x
$$

and

$$
\lim _{n \rightarrow \infty} \sum_{j=1}^{n}\left|w\left(x_{n j}\right)\right| f\left(x_{n j}\right)=\int_{-1}^{1}|k(x)| f(x) d x .
$$

In the next two sections, we consider the construction of product integration rules of type (1), for functions $f(x)$ with a first-order real pole or a pair of first-order complex conjugate poles, i.e., of the form

$$
f(x)=\frac{\bar{f}(x)}{x+u} \text { or } f(x)=\frac{\bar{f}(x)}{(x+\delta)^{2}+\varepsilon^{2}},
$$

with $u$ and $-\delta+i \varepsilon$ outside, but close, to the interval of integration $(a, b)$. To simplify the notation we will take $\delta=0$ in the following, without any loss of generality. Indeed, when $\delta \neq 0$, it is sufficient to shift the interval of integration or replace $\pm i \varepsilon$ in the expressions we give by $-\delta \pm i \varepsilon$.

Rules for integrals of this type have been proposed in [1, Section 2.12.5.1]. A special class of such rules has been explicitly constructed in [11]; however, these latter formulas require the evaluation of $\bar{f}(x)$ at the poles. Since, in our case, the complex poles appear in conjugate pairs, we want to construct quadratures that incorporate these pairs into the coefficients of the rules and require the evaluation of $\bar{f}(x)$ only at points of the (real) interval of integration.

Note that integrals of the form

$$
\int_{a}^{b} k(x) \frac{f(x)}{g(x)} d x
$$

with $g(x)=\prod_{i=1}^{r}\left(x-u_{i}\right) \prod_{j=1}^{s}\left(\left(x+\delta_{j}\right)^{2}+\varepsilon_{j}^{2}\right)$, can be easily reduced to linear combinations of integrals that involve functions of the form (5). 
In our discussion, we assume that we already have, or know how to compute, the product rule (1), and want to construct the new one,

$$
\int_{a}^{b} \bar{k}(x) f(x) d x \doteq \sum_{j=1}^{n} w_{n j}(\bar{k}) f\left(x_{n j}\right),
$$

where

$$
\bar{k}(x)=\frac{k(x)}{x+u} \quad \text { or } \quad \bar{k}(x)=\frac{k(x)}{x^{2}+\varepsilon^{2}},
$$

i.e., express the new coefficients $\left\{w_{n j}(\bar{k})\right\}$ of $(6)$ in terms of the "old" coefficients $\left\{w_{n j}(k)\right\}$ in (1).

Formula (6) is precisely of type (1) with the new kernel $\bar{k}(x)$ defined by (7). Hence, the convergence results reported in Theorem 1 apply also to this new situation.

In Section 4, we consider the case of integrals, over infinite intervals, of meromorphic functions with an infinite number of pairs of first-order complex conjugate poles, for example,

$$
\int_{0}^{\infty} \frac{x}{e^{x}-1} f(x) d x
$$

and use suitable formulas of type (6) to construct accurate approximations. We remark that the case of a finite interval of integration (and infinitely many poles) is similar to that of a finite number of poles, since only those close to the interval will affect significantly the accuracy of a quadrature rule, if the latter does not take them into account properly.

2. Computation of the Coefficients $w_{n j}(\bar{k})$. The case of a single real pole has already been treated in connection with the evaluation of Cauchy principal value integrals; see, for example, [13]. Indeed, the formulas presented there hold also in cases where the pole is outside the interval of integration. Here we recall the formula that establishes the connection between the weights of (1) and (6).

Assume that the nodes $\left\{x_{n j}\right\}$ are the (real) zeros of a (monic) polynomial $P_{n}(x)$ of degree $n$ that satisfies a three-term recurrence relation

$$
\begin{gathered}
P_{-1}(x)=0, \quad P_{0}(x)=1, \\
P_{i+1}(x)=\left(x-a_{i}\right) P_{i}(x)-b_{i} P_{i-1}(x), \quad i=0,1, \ldots .
\end{gathered}
$$

Define

$$
Q_{i}(z)=\int_{a}^{b} k(x) \frac{P_{i}(x)}{x-z} d x, \quad i=0,1, \ldots
$$

It is not difficult to obtain the relations

$$
\begin{gathered}
Q_{-1}(z)=0, \quad Q_{0}(z)=\int_{a}^{b} \frac{k(x)}{x-z} d x, \\
Q_{i+1}(z)=\left(z-a_{i}\right) Q_{i}(z)-b_{i} Q_{i-1}(z)+m_{i}, \quad i=0,1, \ldots,
\end{gathered}
$$

where $m_{i}$ are the modified moments in (2). For $z \in(a, b)$ we define $Q_{i}(z)$ in the Cauchy principal value sense. Note that when $\left\{P_{i}(x)\right\}$ is a system orthogonal in $(a, b)$ with respect to $k(x)$ we have $m_{i}=0, i \geqslant 1$. 
For the coefficients $w_{n j}(\bar{k})$ of the new formula (6), when $\bar{k}(x)=k(x) /(x+u)$, we have (see [13]):

$$
\begin{aligned}
w_{n j}(\bar{k}) & =\frac{1}{x_{n j}+u}\left[w_{n j}(k)-\frac{Q_{n}(-u)}{P_{n}^{\prime}\left(x_{n j}\right)}\right] \\
& =\frac{w_{n j}(k)}{x_{n j}+u}\left[1-\frac{Q_{n}(-u)}{Q_{n}\left(x_{n j}\right)}\right], \quad j=1, \ldots, n .
\end{aligned}
$$

To obtain the second expression for $w_{n j}(\bar{k})$, we need to recall that

$$
w_{n j}(k)=Q_{n}\left(x_{n j}\right) / P_{n}^{\prime}\left(x_{n j}\right) .
$$

When $\bar{k}(x)=k(x) /\left(x^{2}+\varepsilon^{2}\right)$, we can proceed in a similar way and express the corresponding new coefficients $w_{n j}(\bar{k})$ in terms of the old ones, $w_{n j}(k)$. To this end, we note first that since

$$
\frac{1}{x^{2}+\varepsilon^{2}}=\frac{1}{2 i \varepsilon}\left[\frac{1}{x-i \varepsilon}-\frac{1}{x+i \varepsilon}\right]
$$

we have

$$
\begin{aligned}
w_{n j}(\bar{k}) & =\frac{1}{P_{n}^{\prime}\left(x_{n j}\right)} \int_{a}^{b} \frac{k(x)}{x^{2}+\varepsilon^{2}} \frac{P_{n}(x)}{x-x_{n j}} d x \\
& =\frac{1}{2 i \varepsilon} \frac{1}{P_{n}^{\prime}\left(x_{n j}\right)}\left[\int_{a}^{b} \frac{k(x)}{x-i \varepsilon} \frac{P_{n}(x)}{x-x_{n j}} d x-\int_{a}^{b} \frac{k(x)}{x+i \varepsilon} \frac{P_{n}(x)}{x-x_{n j}} d x\right] .
\end{aligned}
$$

Furthermore, using

$$
\frac{1}{(x \pm i \varepsilon)\left(x-x_{n j}\right)}=\frac{1}{\mp i \varepsilon-x_{n j}}\left[\frac{1}{x \pm i \varepsilon}-\frac{1}{x-x_{n j}}\right],
$$

we obtain

$$
w_{n j}(\bar{k})=\frac{1}{\left(x_{n j}^{2}+\varepsilon^{2}\right) P_{n}^{\prime}\left(x_{n j}\right)}\left[Q_{n}\left(x_{n j}\right)-\frac{Q_{n}(i \varepsilon)\left(x_{n j}+i \varepsilon\right)-Q_{n}(-i \varepsilon)\left(x_{n j}-i \varepsilon\right)}{2 i \varepsilon}\right] .
$$

Finally, introducing the real quantities

$$
T_{j}(\varepsilon ; x)=\frac{1}{2 i \varepsilon}\left|\begin{array}{ll}
Q_{j}(i \varepsilon) & (x-i \varepsilon) \\
Q_{j}(-i \varepsilon) & (x+i \varepsilon)
\end{array}\right|=\int_{a}^{b} k(t) \frac{(t+x) P_{j}(t)}{t^{2}+\varepsilon^{2}} d t,
$$

we find

$$
\begin{aligned}
w_{n j}(\bar{k}) & =\frac{1}{x_{n j}^{2}+\varepsilon^{2}}\left[w_{n j}(k)-\frac{T_{n}\left(\varepsilon ; x_{n j}\right)}{P_{n}^{\prime}\left(x_{n j}\right)}\right] \\
& =\frac{w_{n j}(k)}{x_{n j}^{2}+\varepsilon^{2}}\left[1-\frac{T_{n}\left(\varepsilon ; x_{n j}\right)}{Q_{n}\left(x_{n j}\right)}\right], \quad j=1, \ldots, n .
\end{aligned}
$$

It remains to determine the quantities $T_{n}\left(\varepsilon ; x_{n j}\right)$, preferably using only real arithmetic. For this purpose, we introduce the new real quantities

$$
R_{j}(\varepsilon ; x)=\frac{1}{2}\left[Q_{j}(i \varepsilon)(x+i \varepsilon)+Q_{j}(-i \varepsilon)(x-i \varepsilon)\right]=\int_{a}^{b} k(t) \frac{\left(t x-\varepsilon^{2}\right) P_{j}(t)}{t^{2}+\varepsilon^{2}} d t
$$


and use relation (9). After some elementary calculation, we obtain

$$
\begin{gathered}
R_{-1}(\varepsilon ; x)=T_{-1}(\varepsilon ; x)=0, \\
R_{0}(\varepsilon ; x)=P_{0}(x)\left[x \int_{a}^{b} \frac{t k(t)}{t^{2}+\varepsilon^{2}} d t-\varepsilon^{2} \int_{a}^{b} \frac{k(t)}{t^{2}+\varepsilon^{2}} d t\right], \\
T_{0}(\varepsilon ; x)=P_{0}(x)\left[x \int_{a}^{b} \frac{k(t)}{t^{2}+\varepsilon^{2}} d t+\int_{a}^{b} \frac{t k(t)}{t^{2}+\varepsilon^{2}} d t\right], \\
R_{j+1}(\varepsilon ; x)=-\varepsilon^{2} T_{j}(\varepsilon ; x)-a_{j} R_{j}(\varepsilon ; x)-b_{j} R_{j-1}(\varepsilon ; x)+m_{j} x, \\
T_{j+1}(\varepsilon ; x)=R_{j}(\varepsilon ; x)-a_{j} T_{j}(\varepsilon ; x)-b_{j} T_{j-1}(\varepsilon ; x)+m_{j},
\end{gathered}
$$

The use of (12) requires knowledge of the modified moments $m_{j}$, as well as the ability to compute the integrals

$$
\int_{a}^{b} \frac{k(t)}{t^{2}+\varepsilon^{2}} d t, \quad \int_{a}^{b} \frac{t k(t)}{t^{2}+\varepsilon^{2}} d t .
$$

In special cases, these can be expressed in closed form. Two important cases are listed below:

(i)

$$
\begin{aligned}
& \int_{a}^{b} \frac{1}{t^{2}+\varepsilon^{2}} d t=\frac{1}{\varepsilon}\left[\operatorname{arctg}\left(\frac{b}{\varepsilon}\right)-\operatorname{arctg}\left(\frac{a}{\varepsilon}\right)\right] \\
& \int_{a}^{b} \frac{t}{t^{2}+\varepsilon^{2}} d t=\frac{1}{2} \ln \left(\frac{b^{2}+\varepsilon^{2}}{a^{2}+\varepsilon^{2}}\right)
\end{aligned}
$$

(ii)

$$
\begin{aligned}
& \int_{0}^{\infty} \frac{e^{-t}}{t^{2}+\varepsilon^{2}} d t=\frac{1}{\varepsilon}[\mathrm{Ci}(\varepsilon) \sin \varepsilon-\operatorname{si}(\varepsilon) \cos \varepsilon] \\
& \int_{0}^{\infty} \frac{t e^{-t}}{t^{2}+\varepsilon^{2}} d t=-\operatorname{Ci}(\varepsilon) \cos \varepsilon-\operatorname{si}(\varepsilon) \sin \varepsilon
\end{aligned}
$$

where $\mathrm{Ci}(x)$ and $\operatorname{si}(x)$ denote the cosine and sine integrals, respectively:

$$
\operatorname{Ci}(x)=-\int_{x}^{\infty} \frac{\cos (t)}{t} d t, \quad \operatorname{si}(x)=-\int_{x}^{\infty} \frac{\sin (t)}{t} d t
$$

The computation of $w_{n j}(\bar{k})$ by means of (10) and (11) requires, in addition, the evaluation of $Q_{n}\left(x_{n j}\right)$ or of $P_{n}^{\prime}\left(x_{n j}\right)$. The first quantity can be computed using the recurrence relationship (9) when it is stable and $Q_{0}\left(x_{n j}\right)$ is available. Stability appears to be assured, for example, when $k(x)$ is the Jacobi weight function (see [4, Section 3.2.3]). Formula (10) requires also the computation of $Q_{n}(-u)$; when $u$ is very close to the interval of integration-the situation which we are interested in - we have observed acceptable stability of (9) in the case of the Jacobi weight function; otherwise, $Q_{n}(-u)$ should be evaluated by the backward recursion proposed by Gautschi in [6].

We expect the stability behavior of the evaluation of $T_{n}\left(\varepsilon ; x_{n j}\right)$ in (11) by means of (12) to be similar to that of $Q_{n}( \pm i \varepsilon)$; for example, in the Jacobi case it appears acceptable when $\varepsilon$ is sufficiently small. If the forward recursion (12) is not sufficiently stable, we use the alternative expression

$$
T_{n}\left(\varepsilon ; x_{n j}\right)=\operatorname{Re}\left(Q_{n}(i \varepsilon)\right)+\operatorname{Im}\left(Q_{n}(i \varepsilon)\right) x_{n j} / \varepsilon,
$$


and evaluate $Q_{n}(i \varepsilon)$ with the (stable) backward algorithm suggested in [6]:

$$
\begin{gathered}
r_{\nu}^{(\nu)}=0, \quad r_{s-1}^{(\nu)}=\frac{b_{s}}{i \varepsilon-a_{s}-r_{s}^{(\nu)}}, \quad s=\nu, \nu-1, \ldots, 1,0, \\
f_{-1}^{(\nu)}=-1, \quad f_{s}^{(\nu)}=r_{s-1}^{(\nu)} f_{s-1}^{(\nu)}, \quad s=0,1, \ldots, n,
\end{gathered}
$$

where $\nu$ is a sufficiently large integer $(>n)$ determined by the prescribed relative accuracy on the approximation $f_{n}^{(\nu)}$ of $Q_{n}(i \varepsilon)$, and the coefficients $a_{s}, b_{s}\left(b_{0}=m_{0}\right)$ are those of recurrence relation (9). Good estimates of $\nu$ for classical weight functions are given in [6].

When (1) is a classical Gaussian rule, from $[21,15.3 .1,15.3 .5,15.3 .6]$ we derive the following useful representation for $P_{n}^{\prime}(x)$ :

$$
\begin{aligned}
& P_{n}^{(\alpha, \beta)^{\prime}}\left(x_{n j}\right)=(-1)^{j-1}\left[d_{n}(\alpha, \beta)\right]^{1 / 2}\left[\left(1-x_{n j}^{2}\right) w_{n j}(k)\right]^{-1 / 2} \quad(\text { Jacobi }), \\
& d_{n}(\alpha, \beta)=2^{\alpha+\beta+1} \frac{\Gamma(n+\alpha+1) \Gamma(n+\beta+1)}{\Gamma(n+1) \Gamma(n+\alpha+\beta+1)}, \\
& L_{n}^{\prime}\left(x_{n j}\right)=(-1)^{j}\left[x_{n j} w_{n j}(k)\right]^{-1 / 2} \quad \text { (Laguerre), } \\
& H_{n}^{\prime}\left(x_{n j}\right)=(-1)^{j-1} \pi^{1 / 4}\left[2^{n+1} n ! / w_{n j}(k)\right]^{1 / 2} \quad \text { (Hermite), }
\end{aligned}
$$

where the zeros $\left\{x_{n j}\right\}$ are arranged in decreasing order in the Jacobi and Hermite cases, and in increasing order in the Laguerre case. In the identities above, it is assumed that the coefficient $k_{n}$ of $x^{n}$ in $P_{n}^{(\alpha, \beta)}(x), L_{n}(x), H_{n}(x)$ is

$$
k_{n}=2^{-n}\left(\begin{array}{c}
2 n+\alpha+\beta \\
n
\end{array}\right), \quad k_{n}=\frac{(-1)^{n}}{n !}, \quad k_{n}=2^{n},
$$

respectively. From the first equation in (11), we then obtain

$$
\begin{gathered}
w_{n j}(\bar{k})=\frac{1}{x_{n j}^{2}+\varepsilon^{2}}\left\{w_{n j}(k)+(-1)^{j}\left[\left(1-x_{n j}^{2}\right) w_{n j}(k) / d_{n}(\alpha, \beta)\right]^{1 / 2}\right. \\
\left.\times k_{n} T_{n}\left(\varepsilon ; x_{n j}\right)\right\} \quad \text { (Jacobi), } \\
\begin{aligned}
w_{n j}(\bar{k})=\frac{1}{x_{n j}^{2}+\varepsilon^{2}}\left\{w_{n j}(k)+(-1)^{n+1-j} / n !\left[x_{n j} w_{n j}(k)\right]^{1 / 2}\right. \\
\left.\times T_{n}\left(\varepsilon ; x_{n j}\right)\right\} \quad \text { (Laguerre), } \\
w_{n j}(\bar{k})=\frac{1}{x_{n j}^{2}+\varepsilon^{2}}\left\{w_{n j}(k)+(-1)^{j} \pi^{-1 / 4}\left[\left(2^{n-1} / n !\right) w_{n j}(k)\right]^{1 / 2}\right.
\end{aligned} \\
\left.\times T_{n}\left(\varepsilon ; x_{n j}\right)\right\} \quad \text { (Hermite). }
\end{gathered}
$$

Note that in the Jacobi case, to avoid the loss of precision caused by the factor $\left(1-x_{n j}^{2}\right)^{1 / 2}$ when $x_{n j}$ is too close to \pm 1 , it is sufficient to use the expression $\left(1-x_{n j}^{2}\right)^{1 / 2}=\sin \left(\arccos \left(x_{n j}\right)\right)$.

Remark. When $k(x)$ in (1) is nonnegative and $\left\{P_{j}(x)\right\}$ is the set of polynomials orthogonal on $(a, b)$ with respect to $k(x)$, Gautschi [5] presents an algorithm for constructing new Gaussian rules associated with the weight functions $\bar{k}(x)$ given in 
(7). The nodes of these new rules, however, depend on the "parameters" $u$ and $\varepsilon$; if $u$ and $\varepsilon$ are to assume several values, a procedure of this type appears costly.

3. Numerical Examples. We have considered the following two integrals:

$$
\begin{array}{ll}
I_{1}=\int_{-1}^{1} \frac{e^{x}}{x^{2}+\varepsilon^{2}} d x, & \varepsilon=1 ., 0.5,0.1,0.01 \\
I_{2}=\int_{0}^{\infty} \frac{e^{-x}}{x^{2}+\varepsilon^{2}} d x, & \varepsilon=2^{k}, k=-2,-1,0,1,2,3,4 .
\end{array}
$$

The calculations have been carried out on a VAX/11-780 with 16 digit double-precision arithmetic.

\section{TABLE 1}

$$
\begin{aligned}
& I_{1}=\int_{-1}^{1} \frac{e^{x}}{x^{2}+\varepsilon^{2}} d x(*) \\
& \varepsilon=1 \text {. } \\
& E_{G L} \\
& I_{1}=0.1795521283093888 \mathrm{E}+1 \\
& \begin{array}{ll}
4 & 6.5 \mathrm{E}-4 \\
8 & 5.8 \mathrm{E}-7
\end{array} \\
& E_{G}(* *) \\
& E_{G M} \\
& 1.1 \mathrm{E}-7 \\
& \text { 9.7 } \mathrm{E}-5 \\
& 5.8 \mathrm{E}-7 \\
& 3.2 \mathrm{E}-14 \\
& 1.1 \mathrm{E}-10 \\
& 4.1 \mathrm{E}-13 \\
& 3.2 \mathrm{E}-14 \\
& 8.8 \mathrm{E}-16 \\
& 6.8 \mathrm{E}-16 \\
& 6.5 \mathrm{E}-16 \\
& \varepsilon=0.5 \\
& E_{G L} \\
& 42.9 \mathrm{E}-2 \\
& I_{1}=0.4893904674627017 \mathrm{E}+1 \\
& E_{G}(* *) \\
& E_{G M} \\
& 9.1 \mathrm{E}-8 \quad 5.0 \mathrm{E}-4 \\
& 6.2 \mathrm{E}-4 \\
& 8.8 \mathrm{E}-16 \\
& 2.8 \mathrm{E}-9 \\
& 5.0 \mathrm{E}-16 \\
& 5.7 \mathrm{E}-16 \\
& 5.8 \mathrm{E}-14 \\
& 2.5 \mathrm{E}-16 \\
& 3.9 \mathrm{E}-16 \\
& \varepsilon=0.1 \\
& I_{1}=0.303030 \\
& E_{G} \\
& E_{G M} \\
& E_{G L} \\
& 0.59 \\
& 3.1 \mathrm{E}-8 \\
& 2.4 \mathrm{E}-3 \\
& 0.32 \\
& 1.2 \mathrm{E}-16 \\
& 6.1 \mathrm{E}-8 \\
& 7.4 \mathrm{E}-2 \\
& 2.1 \mathrm{E}-16 \\
& 32 \quad 3.1 \mathrm{E}-3 \\
& \varepsilon=0.01 \\
& E_{G L} \\
& \begin{array}{c}
I_{1}=0.3131720562393341 \mathrm{E}+3 \\
E_{G} \quad E_{G M}
\end{array} \\
& 0.96 \\
& 3.6 \mathrm{E}-9 \\
& 3.5 \mathrm{E}-3
\end{aligned}
$$$$
n
$$$$
16
$$$$
32
$$$$
16 \quad 2.8 \mathrm{E}-7
$$$$
32
$$$$
8
$$$$
64 \quad 5.3 \mathrm{E}-6
$$

$8 \quad 0.92$

$16 \quad 0.84$

$32 \quad 0.69$

$64 \quad 0.43$

(*) $E_{(i L}, E_{G i}, E_{G M}$ below indicates the relative accuracy obtained by the respective formula $G L_{n}, G_{n}$, $G M_{n}$.

$\left({ }^{* *}\right)$ Gautschi in his paper [5] points out the strong tendency of his procedure to become unstable when the poles move away from the interval and suggests a variation of his approach; nevertheless, we have used the algorithm presented in [5]. 
TABLE 2

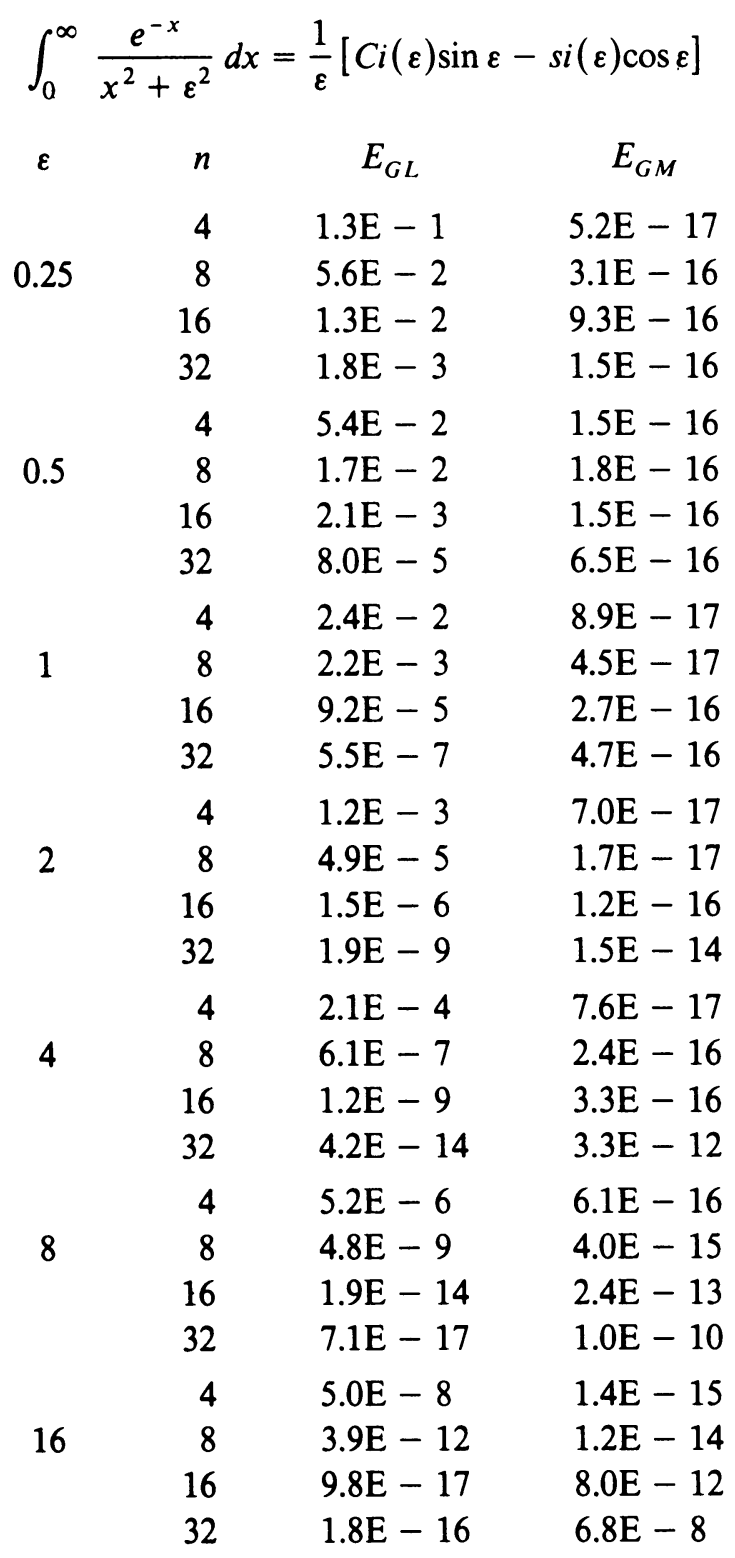

Integral $I_{1}$ has been estimated by applying to it a Gauss-Legendre rule $\left(G L_{n}\right)$, a Gaussian rule associated with $\bar{k}(x)=1 /\left(x^{2}+\varepsilon^{2}\right)\left(G_{n}\right)$, constructed with the algorithm given in [5], and our formula (6) $\left(G M_{n}\right)$ where $T_{n}\left(\varepsilon ; x_{n j}\right)$ has been computed using the forward recurrence relation (12) when $\varepsilon=0.1,0.01$, and by (14) (together with Gautschi's algorithm (15) to compute $\left.Q_{n}(i \varepsilon)\right)$ when $\varepsilon=0.5,1$. Some of the results we have obtained are listed in Table 1.

To approximate integral $I_{2}$ we have used a Gauss-Laguerre rule $\left(G L_{n}\right)$, and our formula (6) $\left(G M_{n}\right)$ (which should give the exact value of $\left.I_{2}\right)$ with the coefficients $w_{n j}(\bar{k})$ given by $(17)$, where $T_{n}\left(\varepsilon ; x_{n j}\right)$ have been obtained from relation (12). In this second case, relation (12) appears to become increasingly unstable as $\varepsilon$ and $n$ 
increase; this phenomenon shows up in some approximations reported in Table 2. If we evaluate $T_{n}\left(\varepsilon ; x_{n j}\right)$ by means of (14), with $Q_{n}(i \varepsilon)$ computed using the backward scheme (15), we have full stability and get an approximation to machine accuracy. This latter algorithm, however, turns out to be a little more expensive in terms of arithmetic operations.

4. Integrals of Functions with Infinitely Many Poles. Recently, integrals of the form

$$
\int_{0}^{\infty} \frac{x}{e^{x}-1} f(x) d x, \quad \int_{0}^{\infty} \frac{1}{e^{x}+1} f(x) d x
$$

were considered by Gautschi and Milovanović [8]. The kernels of both integrals have an infinite number of pairs of first-order complex conjugate poles: $\pm i 2 m \pi, m=1$, $2, \ldots$, in the first integral, and $\pm i(2 m-1) \pi, m=1,2, \ldots$, in the second.

Approximations to integrals of the type above can be constructed using formulas of type (6). To show how this can be done, consider, for example, the first integral, and rewrite it as follows:

$$
I(f)=\int_{0}^{\infty} e^{-x} \frac{x}{1-e^{-x}} f(x) d x .
$$

Recalling the Mittag-Leffler theorem (see, for example, [10, pp. 655 and 660]), we obtain a partial fraction expansion of the function $x /(1-\exp (-x))$,

$$
\frac{x}{1-e^{-x}}=-\frac{1}{2} x+1+2 x^{2} \sum_{m=1}^{\infty} \frac{1}{x^{2}+4 \pi^{2} m^{2}},
$$

where the series converges uniformly on every bounded set not containing any of the poles. The function

$$
\frac{x}{1-e^{-x}}-2 x^{2} \sum_{m=1}^{N} \frac{1}{x^{2}+4 \pi^{2} m^{2}}=-\frac{1}{2} x+1+2 x^{2} \sum_{m=N+1}^{\infty} \frac{1}{x^{2}+4 \pi_{f}^{2} m^{2}}
$$

is analytic on any bounded set not containing the poles $\pm i 2 m \pi, m=N+1$, $N+2, \ldots$ This suggests that we express $I(f)$ in the form

$$
\begin{aligned}
I(f)= & \int_{0}^{\infty} e^{-x}\left[\frac{x}{1-e^{-x}}+\sum_{m=1}^{N} \frac{8 \pi^{2} m^{2}}{x^{2}+4 \pi^{2} m^{2}}\right] f(x) d x \\
& -\sum_{m=1}^{N} 8 \pi^{2} m^{2} \int_{0}^{\infty} e^{-x} \frac{f(x)}{x^{2}+4 \pi^{2} m^{2}} d x
\end{aligned}
$$

and apply the $n$-point Gauss-Laguerre rule to the first integral, and the respective formula of type (6), with the weights $w_{n j}(\bar{k})$ given by (17), to the second integral. Note that one can subtract out one pair of poles at a time, until the influence of the remaining ones is sufficiently small; in this case, having already computed

$$
I_{0}(f)=\sum_{j=1}^{n} v_{n j}^{(0)} f\left(x_{n j}\right), \quad v_{n j}^{(0)}=w_{n j}(k) \frac{x_{n j}}{1-e^{-x_{n j}}},
$$


one determines successively, for $m=1,2, \ldots, N$,

$$
\begin{aligned}
& I_{m}(f)=\sum_{j=1}^{n} v_{n j}^{(m)} f\left(x_{n j}\right), \\
& v_{n j}^{(m)}=v_{n j}^{(m-1)}+\frac{8 m^{2} \pi^{2}}{x_{n j}^{2}+4 m^{2} \pi^{2}}(-1)^{n-j}\left(x_{n j} w_{n j}(k)\right)^{1 / 2} T_{n}\left(2 m \pi ; x_{n j}\right),
\end{aligned}
$$

where $T_{n}\left(2 m \pi ; x_{n j}\right)$ is given by (14) and $Q_{n}(i 2 m \pi)$ is computed using (15).

In Tables 3 and 4 below we list some of the results we have obtained. $E_{G L}$ and $E_{G M}$ denote the relative errors obtained respectively by the $n$-point Gauss-Laguerre rule and the corresponding scheme (19).

All the numerical results presented in Tables 3, 4 have been obtained on a VAX/11-780, working with 16 digit double-precision arithmetic.

We must, however, remark that the procedure we have proposed appears effective only when the accuracy of the chosen basic rule (1) is bounded mainly by the presence of poles, and not by the too low number of nodes in the formula. Indeed, it may happen that the limitation of precision in the approximation obtained with the

\section{TABLE 3}

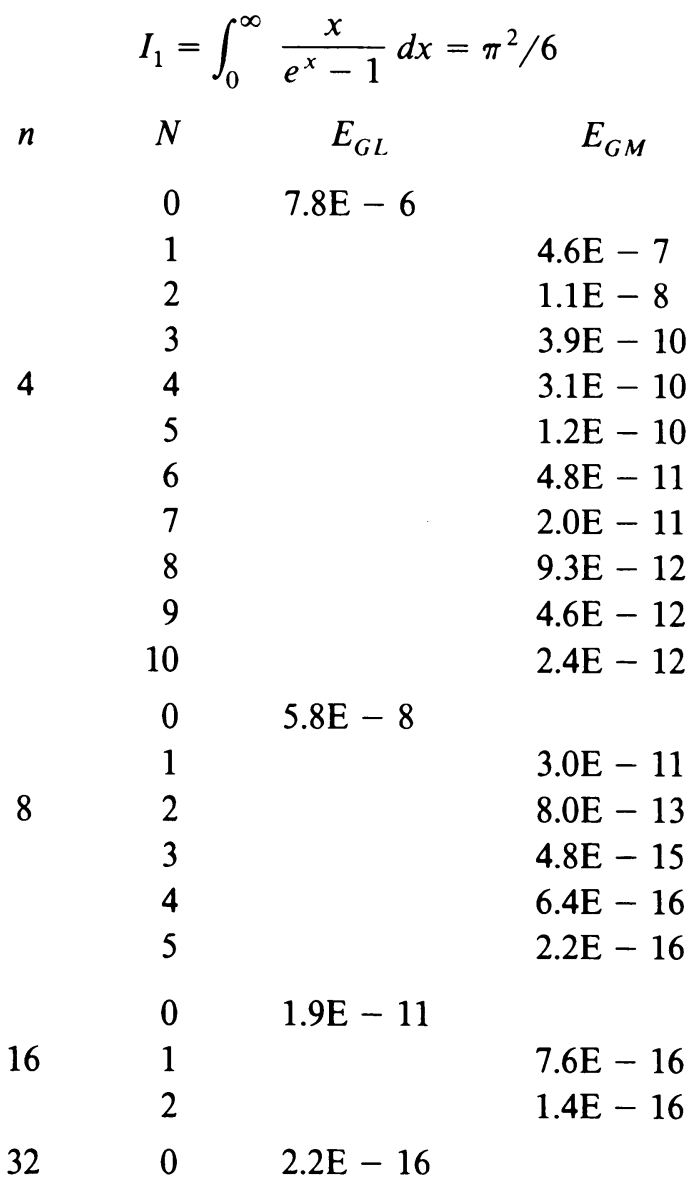


TABLE 4

$\begin{array}{cccc}I_{2}=\int_{0}^{\infty} \frac{x}{e^{x}-1} x^{10} d x=(2 \pi)^{12} 691 / 65520 \\ n & N & E_{G L} & E_{G M} \\ & 0 & 7.9 \mathrm{E}-11 & \\ 16 & 1 & & 2.5 \mathrm{E}-12 \\ & 2 & & 6.0 \mathrm{E}-14 \\ & 3 & & 1.1 \mathrm{E}-15 \\ 32 & 0 & 8.9 \mathrm{E}-16 & \end{array}$

chosen quadrature is caused mainly by the low number of nodes, and the removal of poles does not produce any improvement. This appears to be the case, e.g., for the integral $I_{2}$ when $n=4,8$ (not shown in Table 4). This phenomenon was also observed when in $I_{2}$ we replaced $x^{10}$ by polynomials of different type, or the infinite interval $(0, \infty)$ by a finite one $(0, b)$ and applied quadratures based on GaussLegendre rules.

Acknowledgment. The author is indebted to F. Neri for his help in the numerical testing.

Dipartimento di Matematica

Politecnico di Torino

Corso Duca degli Abruzzi, 24

10129 Torino, Italy

1. P. J. Davis \& P. Rabinowitz, Methods of Numerical Integration, Academic Press, New York, 1975.

2. D. Elliotr \& D. F. PAget, "Product integration rules and their convergence," BIT, v. 16, 1976, pp. $32-40$.

3. D. Elliott \& D. F. PAget, “The convergence of product integration rules," BIT, v. 18, 1978, pp. $137-141$

4. W. Gautschi, "A survey of Gauss-Christoffel quadrature formulae," in E. B. Christoffel, The Influence of his Work in Mathematics and the Physical Sciences; International Christoffel Symposium (P. Butzer and F. Fehér, eds.), Birkhäuser, Basel, 1981, pp. 72-147.

5. W. Gautschi, "An algorithmic implementation of the generalized Christoffel theorem," in Numerical Integration (G. Hämmerlin, ed.), ISNM 57, Birkhäuser, Basel, 1982, pp. 89-106.

6. W. GaUTSCHI, "Minimal solution of three-term recurrence relations and orthogonal polynomials," Math. Comp., v. 36, 1981, pp. 547-554.

7. W. GautschI, “Discrete approximations to spherically symmetric distributions," Numer. Math., v. 44, 1984, pp. 53-60.

8. W. Gautschi \& G. V. Milovanovic, "Gaussian quadrature involving Einstein and Fermi functions with an application to summation of series," Math. Comp., v. 44, 1985, pp. 177-190.

9. I. S. Gradshteyn \& I. M. RyzhiK, Tables of Integrals, Series, and Products, Academic Press, New York, 1965.

10. P. Henrici, Applied and Computational Complex Analysis, Vol. I, Wiley, New York, 1974.

11. F. G. Lether, "Modified quadrature formulas for functions with nearby poles," J. Comput. Appl. Math., v. 3, 1977, pp. 3-9.

12. G. G. LoRENTZ, Approximation of Functions, Holt, Rinehart and Winston, New York, 1966.

13. G. Monegato, "The numerical evaluation of one-dimensional Cauchy principal value integrals," Computing, v. 29, 1982, pp. 337-354.

14. I. H. SLOAN, “On the numerical evaluation of singular integrals," $B I T$, v. 18, 1978, pp. 91-102.

15. I. H. Sloan, "On choosing the points in product integrations," J. Math. Phys., v. 21, 1980, pp. 1032-1039. 
16. I. H. SloAN \& W. E. Smith, "Product integration with Clenshaw-Curtis and related points: convergence properties," Numer. Math., v. 30, 1978, pp. 415-428.

17. I. H. SlOAN \& W. E. SMITH, "Product integration with the Clenshaw-Curtis points: implementation and error estimates," Numer. Math., v. 34, 1980, pp. 387-401.

18. I. H. SloAN \& W. E. SMITh, "Properties of interpolatory product integration rules," SIAM J. Numer. Anal., v. 19, 1982, pp. 427-442.

19. I. H. SloAN \& W. E. SMith, "Product integration rules based on the zeros of Jacobi polynomials," Math. Comp., v. 37, 1980, pp. 1-13.

20. W. E. SMith, I. H. SloAN \& A. H. OpIE, "Product integration over infinite intervals I. Rules based on the zeros of Hermite polynomials," Math. Comp., v. 40, 1983, pp. 519-535.

21. G. Szego, Orthogonal Polynomials, Amer. Math. Soc. Colloq. Publ., vol. 23, Amer. Math. Soc., Providence, R. I., 1975. 